\title{
Risk of Gestational Diabetes Among South Asian Immigrants Living in New Jersey—a Retrospective Data Review
}

\author{
Acharyya Sanchalika $^{1} \cdot$ Janevic Teresa $^{2}$
}

Received: 14 October 2014 / Revised: 23 February 2015 / Accepted: 27 February 2015 / Published online: 19 March 2015

(C) W. Montague Cobb-NMA Health Institute 2015

\begin{abstract}
Background We aimed to identify differences in distribution of risk factors, prevalence, and complications of gestational diabetes (GD) among South Asian (SA) immigrant women, separately for immigrants from India, Bangladesh, Pakistan, and Sri Lanka, relative to US-born non-Hispanic whites (NHW) living in New Jersey.

Method We used NJ birth certificate data linked to hospitalization data from 1999 to $2002(n=327,069)$. We compared the distribution of risk factors among these groups using chisquared test and calculated adjusted odds of GD for SA groups compared to NHW using logistic regression. Among women with GD, we further analyzed the odds of complications for SA groups relative to whites.

Results Sri Lankans were more likely to be of advanced maternal age. Pakistanis and Bangladeshis were more likely to start prenatal care late. Bangladeshis had the highest adjusted odds of GD (aOR $=4.3 ; 95 \%$ confidence interval $(\mathrm{CI}) 3.5$ 5.3), followed by Indians ( $\mathrm{aOR}=3.9 ; 95 \%$ CI 3.7-4.2), Sri Lankans (aOR=3.6; $95 \%$ CI 2.4-5.8), and Pakistanis (aOR= $3.4 ; 95 \%$ CI 2.9-3.8) relative to NHW. Among women with GD, South Asian groups had lower odds of preterm birth and higher odds of having a small for gestational age infant than whites.

Discussion This study provided evidence of disproportionate risk of GD among four SA groups living in NJ.
\end{abstract}

Acharyya Sanchalika

sanchalika.acharyya@duke-nus.edu.sg

1 Centre for Quantitative Medicine, DUKE NUS Graduate Medical School, 20 College Road, Level 6, Singapore 169856, Singapore

2 Department of Epidemiology, Rutgers School of Public Health, Piscataway, NJ 08854, USA
Keywords South Asian · Gestational diabetes · Risk factors · Pregnancy complications

\section{Introduction}

Gestational diabetes is defined as the reduced sensitivity to insulin, detected first time during pregnancy, complicating $1-14 \%$ of all pregnancies [1]. Gestational diabetes is typically diagnosed around 24th week of gestation and may cause serious to fatal obstetric complications and fetal morbidities if left untreated $[2,3]$. Previous studies have demonstrated ethnic heterogeneity in the prevalence of gestational diabetes. According to the Northern California Kaiser Permanente study between 1991 and 2000, the increment in prevalence of gestational diabetes was highest among Asians and AfricanAmericans as compared to Hispanic, and non-Hispanic whites [3]. A study conducted by Thorpe et al. (2005) in New York City documented the highest prevalence of gestational diabetes among South-Central Asian (11.1\%) in 2001 and maximum increase among Mexican (96\%), followed by SouthCentral Asian (95\%), and non-Hispanic black women (82\%), from the year 1990 to 2001 [4]. An analysis of birth records in New York between 1995 and 2003 further demonstrated the largest relative risk of gestational diabetes among South-Central Asians (RR 4.7; $95 \%$ confidence interval (CI) 4.6-4.9) as compared to other ethnic groups [5]. Several other studies [6-10] from around the world identified similar trend in increased prevalence of gestational diabetes among South Asian immigrants. A limitation of previous research, however, is combining South Asian groups with distinct socioeconomic and migration profiles, such as Indians and Bangladeshis, into an overarching category. Further, the distribution of known risk factors among these separate groups has not previously been explored. 
Given the fact that New Jersey is home to more than 300, 000 immigrants with roots in India, Bangladesh, Pakistan, and Sri Lanka, comprising the third largest population of South Asians [11] in the USA, it is an ideal setting in which to study the maternal health of South Asians. Because no previous study has examined the maternal health of South Asian diaspora living in New Jersey, we undertook this current study with the aim to identify any disparity in prevalence, distribution of risk factors, and complications of gestational diabetes among specific South Asian immigrant groups, in comparison to non-Hispanic US-born white women.

\section{Methods}

\section{Data Source}

We used New Jersey electronic birth certificate data cross-sectionally linked to hospitalization data for the years 1999-2002, requested and obtained from New Jersey Department of Health and Senior Services (NJDHSS), for this secondary data analysis $(n=549$, $300)$. The dataset included information on demographics, and complications during pregnancy, delivery, and post-delivery of all women admitted for child birth during that period. All births reports were crosssectionally linked to mother's data using the mother's and infant's unique personal identification number. We limited our analysis only to singleton live births between January 1, 1999 and December 30, 2002. For analyzing pregnant women with gestational diabetes, we further generated a subset of this dataset including only those without a history of diabetes prior to first delivery. Pregnancy complication variables were created from International Classification of Diseases, Ninth Revision, Clinical Modification (ICD-9) codes. We used "country of birth" to determine the specific immigrant groups originating from four South Asian countries namely India, Bangladesh, Pakistan and Sri Lanka. Since the majority of the South Asian population living in New Jersey are foreign born [12], our study hopes to capture most of the women belonging to this immigrant group. We also kept data from US-born non-Hispanic white women as a comparison group. Any missing value for diagnosis of gestational diabetes and infant data missing a maternal identifier were dropped. In addition, we also deleted any observation with missing values for health insurance status. The final dataset used for this study had 327,069 observations. We conducted all statistical analyses using SAS statistical package version 9.2. This study was approved by the Institutional Review Board of University of Medicine and Dentistry of New Jersey.

\section{Exposure, Outcome, and Covariates}

The main exposure variable of this study was maternal country of birth. If the mother's birth place was recorded as any city or state of USA and her race were reported as non-Hispanic white, the mother was coded as "non-Hispanic white" in the dataset. Similarly, if the mother's birth place was recorded as any region, city or state of India, Bangladesh, Pakistan, and Sri-Lanka, the mother was coded as "Indian," "Bangladeshi," "Pakistani," and "Sri Lankan," respectively. Women from these four countries were analyzed separately. The diagnosis of gestational diabetes was performed following ICD-9 codes 64881 and 64882. Consistent with previous research, we considered women who had an indication of gestational diabetes on either the birth certificate or the hospital discharge data (or both) as cases with gestational diabetes and those who had no indication on either source as women without gestational diabetes. This combined method of case ascertainment for gestational diabetes has been proven to be much superior to birth certificates alone in the reporting of gestational diabetes $[5,13]$. We excluded women diagnosed with established diabetes following ICD-9 codes (25000-25093, 36201, and 64801-64802) from the analyses since they were not at risk of gestational diabetes.

Sociodemographic characteristics consisted of patient reported maternal age (less than 25 years, 25-29 years, 30-34 years, 35-39 years, and 40 years or more), maternal education (less than 12 years/high school, 12 years/high school, 13-15 years/some college, and $\geq 16$ years or more/completion of college), initiation of prenatal care (first, second, or third trimester), number of previous live births or parity (0/nulliparous, 1, 2, 3, and more than 3 ), and health insurance coverage status (covered or not covered). We created a new variable for prenatal care by combining categories; if the prenatal care was initiated during first and second trimester, the new variable was coded as "timely initiation of prenatal care." Otherwise, we coded the variable as "late prenatal care." Health-related behavior included use of any number of tobacco products and any amount of alcohol consumption during pregnancy and was coded as binary variable with levels yes or no.

Pregnancy outcomes of interest related to gestational diabetes included preterm birth (defined as gestational age less than 37 weeks), small for gestational age tenth and fifth percentiles, and large for gestational age defined as child's weight at delivery more than $4000 \mathrm{~g}$ [14]. Infant's birth weight and gestational age were ascertained from birth certificate data. The gestational age at delivery was ascertained by clinical estimate using prenatal ultrasound instead of last menstrual dating method [15]. 


\section{Statistical Analysis}

We compared the percent distributions of maternal age, education, health insurance coverage, smoking, drinking, parity, and initiation of prenatal care across the five groups using Mantel-Haenszel chi-squared tests. We estimated the prevalence of gestational diabetes for all five groups separately and then used Mantel-Haenszel chi-squared test to check whether the prevalences were significantly different between South Asian groups and non-Hispanic whites. We then estimated the odds ratios, both unadjusted and adjusted for potential confounders, for gestational diabetes comparing South Asian groups and non-Hispanic whites using logistic regression. We selected maternal age at delivery, maternal education, health insurance coverage, parity, initiation of prenatal care, and smoking and drinking habits as potential confounders based on related previous literature. Among the women diagnosed with gestational diabetes, we compared the odds of subsequent perinatal complications for South Asian groups with that of US-born non-Hispanic white women using logistic regression models. All statistical analyses were carried out in SAS 9.2 (SAS Institute, Cary, NC) and at a $5 \%$ significance level.

\section{Results}

In our study sample, $4 \%$ of the pregnant women were diagnosed with gestational diabetes $(n=12998)$. When stratified by ethnic group, Sri Lankans had the highest prevalence of gestational diabetes $(12.5 \%)$, followed by Bangladeshis (12.4\%), Indians (11.0\%), and Pakistanis (10.4\%). In contrast, only $3.5 \%$ of US-born non-Hispanic white women were diagnosed with gestational diabetes (Fig. 1a). The differences were found to be statistically significant in Mantel-Haenszel chi-squared test at $5 \%$ significance level ( $p$ value $<0.0001)$.

In terms of the distribution of risk factors across five comparison groups, we found that Indians and Sri Lankan women had higher levels of formal education (16 years or more) than others ( $p$ value $<0.0001)$ and Sri Lankan women were more likely to be aged 30 years or older than other groups ( $p$ value $<$ 0.0001). Pakistanis and Bangladeshis were more likely to have three or more children and less likely to be covered by health insurance than others ( $p$ value $<0.0001)$. The use of tobacco $(p$ value $<0.0001)$ and alcohol $(p$ value $=0.0046)$ was highest among non-Hispanic white women. Initiation of prenatal care in third trimester was highest among Pakistani women followed by Bangladeshis $(p$ value $<0.0001)$ (Table 1).

After adjusting for maternal age, education, parity, health insurance coverage, reported smoking and drinking habits, and timely initiation of prenatal care, the odds of gestational diabetes for Bangladeshi women was four times the odds of that among non-Hispanic whites $(\mathrm{aOR}=4.3$, $95 \%$ CI 3.55.3). Indians, Sri Lankans, and Pakistanis had odds of 3.9 (95\% CI 3.7-4.2), 3.7 (95\% CI 2.4-5.8), and 3.4 (95\% CI 2.9-3.8), respectively, when compared to non-Hispanic whites after adjusting for potential covariates (Fig. 1b).

After adjustment for maternal age, education, health insurance coverage, parity, and smoking and drinking habits, Bangladeshi $(\mathrm{aOR}=3.7,95 \%$ CI 2.3-6.0), Indian $(\mathrm{aOR}=2.7$, $95 \%$ CI 2.3-3.2), and Pakistani $(\mathrm{aOR}=2.2$, $95 \%$ CI 1.6 3.0) women diagnosed with gestational diabetes had significantly higher adjusted odds of having a small for gestational age at the tenth percentile (SGA 10th) infant than nonHispanic white women (Table 2). Bangladeshi $(\mathrm{aOR}=2.4$, $95 \%$ CI 1.2-5.1) and Indian $(\mathrm{aOR}=2.9,95 \%$ CI 2.3-3.5) women with gestational diabetes were found to have the
Fig. 1 Maternal ethnicity versus a prevalence of gestational diabetes and $\mathbf{b}$ adjusted odds ratio estimates with $95 \%$ CI

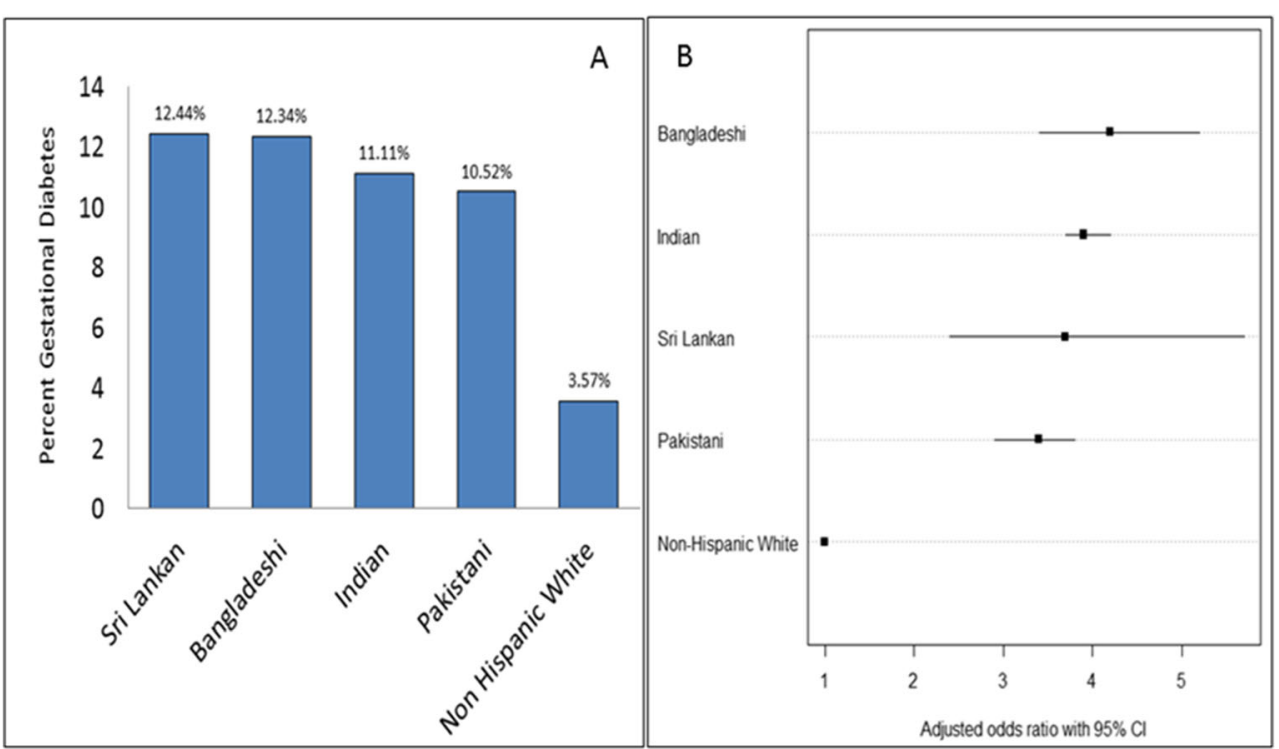


Table 1 Demographics of pregnant women of US-born non-Hispanic white origin and four groups of South Asian immigrants-NJ births, 1999-2002

\begin{tabular}{|c|c|c|c|c|c|c|}
\hline \multirow{2}{*}{$\begin{array}{l}\text { Characteristics frequency } \\
(\%)\end{array}$} & \multicolumn{6}{|c|}{ Maternal country of birth/ethnicity $(n=327,069)$} \\
\hline & $\begin{array}{l}\text { Indian } \\
(n=14612)\end{array}$ & $\begin{array}{l}\text { Bangladeshi } \\
(n=833)\end{array}$ & $\begin{array}{l}\text { Pakistani } \\
(n=2924)\end{array}$ & $\begin{array}{l}\text { Sri-Lankan } \\
(n=192)\end{array}$ & $\begin{array}{l}\text { Non-Hispanic white } \\
(n=308508)\end{array}$ & $\begin{array}{l}p \\
\text { value }\end{array}$ \\
\hline \multicolumn{7}{|l|}{ Maternal age (years) } \\
\hline$<25$ & $1659(11.3)$ & $226(27.1)$ & $563(19.2)$ & $15(7.8)$ & $73,514(23.8)$ & \multirow[t]{5}{*}{$<0.001$} \\
\hline $25-29$ & $6836(46.8)$ & $308(37.0)$ & $1023(34.9)$ & $48(25.0)$ & $70,480(22.8)$ & \\
\hline $30-34$ & $4772(32.6)$ & $201(24.1)$ & $864(29.5)$ & $69(35.9)$ & $99,148(32.1)$ & \\
\hline $35-39$ & $1189(8.1)$ & $76(9.1)$ & $402(13.7)$ & $49(25.5)$ & $54,256(17.6)$ & \\
\hline$\geq 40$ & $156(1.1)$ & $22(2.6)$ & $72(2.4)$ & $11(5.7)$ & $11,102(3.6)$ & \\
\hline \multicolumn{7}{|l|}{ Maternal education (years) } \\
\hline$<12$ & $372(2.6)$ & $196(24.8)$ & $317(11.3)$ & $10(5.3)$ & $30,758(10.1)$ & \multirow[t]{4}{*}{$<0.001$} \\
\hline 12 & $1144(7.9)$ & $252(31.9)$ & $712(25.5)$ & $32(16.8)$ & $89,678(29.5)$ & \\
\hline $13-15$ & $3137(21.8)$ & $149(18.8)$ & $707(25.3)$ & $35(18.4)$ & $70,517(23.2)$ & \\
\hline 16 or more & 9734 (67.6) & $194(24.5)$ & $1056(37.8)$ & $113(59.5)$ & $112,612(37.1)$ & \\
\hline \multicolumn{7}{|l|}{ Smoking } \\
\hline Yes & $20(0.1)$ & $1(0.1)$ & $20(0.7)$ & $0(0.0)$ & $39,042(12.7)$ & \multirow[t]{2}{*}{$<0.001$} \\
\hline No & $14,578(99.7)$ & $831(99.7)$ & $2891(98.8)$ & $192(100.0)$ & $268,170(86.9)$ & \\
\hline \multicolumn{7}{|l|}{ Drinking } \\
\hline Yes & $14(0.1)$ & $0(0.00)$ & $3(0.1)$ & $0(0.0)$ & $5129(1.6)$ & \multirow[t]{2}{*}{0.0048} \\
\hline No & $14,584(99.8)$ & $832(99.8)$ & $2907(99.4)$ & $192(100.0)$ & $301,805(97.8)$ & \\
\hline \multicolumn{7}{|l|}{ Health insurance } \\
\hline Not covered & $817(5.6)$ & $85(10.2)$ & $498(17.0)$ & $15(7.8)$ & $11,437(3.7)$ & \multirow[t]{2}{*}{$<0.001$} \\
\hline Covered & $13,795(94.4)$ & $748(89.8)$ & $2426(82.9)$ & $177(92.2)$ & $297,071(96.3)$ & \\
\hline \multicolumn{7}{|c|}{ No. of previous live births/parity } \\
\hline Nulliparous & $7847(53.7)$ & $327(39.3)$ & $1036(35.4)$ & $86(44.8)$ & $121,418(39.5)$ & \multirow[t]{5}{*}{$<0.001$} \\
\hline 1 & $5594(38.3)$ & $276(33.1)$ & $911(31.2)$ & $71(36.9)$ & $102,307(33.1)$ & \\
\hline 2 & $1003(6.9)$ & $133(15.9)$ & $600(20.5)$ & $29(15.1)$ & $52,749(17.1)$ & \\
\hline 3 & $141(0.9)$ & $57(6.8)$ & $254(8.7)$ & $6(3.1)$ & $19,838(6.4)$ & \\
\hline$>3$ & $27(0.2)$ & $40(4.8)$ & $123(4.2)$ & $0(0.0)$ & $12,196(3.9)$ & \\
\hline \multicolumn{7}{|l|}{ Initiation of prenatal care } \\
\hline None & $30(0.2)$ & $6(0.7)$ & $25(0.8)$ & $0(0.0)$ & $4238(1.4)$ & \multirow[t]{4}{*}{$<0.001$} \\
\hline First trimester & $12,372(84.7)$ & $563(67.6)$ & $2040(69.8)$ & $161(83.8)$ & $247,396(80.2)$ & \\
\hline Second trimester & $1568(10.7)$ & $196(23.5)$ & $528(18.1)$ & $18(9.4)$ & $37,962(12.3)$ & \\
\hline Third trimester & $642(4.4)$ & $68(8.2)$ & $331(11.3)$ & $13(6.8)$ & $18,912(6.1)$ & \\
\hline
\end{tabular}

adjusted odds of having SGA 5th percentile infant more than two times the odds among white women with gestational diabetes. However, SA women across all ethnic groups with gestational diabetes had lower odds of having a baby weighing more than $4000 \mathrm{~g}$ than white women with gestational diabetes. We dropped the Sri Lankan women from this set of analysis due to their small numbers.

\section{Discussion}

In this large population-based cross-sectional study, we documented differential risks of gestational diabetes for South Asian immigrants in comparison to US-born non-Hispanic white women using data on singleton births for the years
1999-2002. Our research has three main findings: (1) Pakistanis and Bangladeshis were more likely to have no health insurance coverage and to have late initiation of prenatal care than other groups; (2) we observed significant higher odds of gestational diabetes among South Asian immigrants relative to US-born non-Hispanic white women; and (3) among women diagnosed with gestational diabetes, the odds of having a small for gestational age infant was higher among South Asian immigrants than non-Hispanic whites.

Our findings contribute to the small body of literature on the risk of gestational diabetes in specific subgroups of South Asian immigrants. We found Bangladeshi women with the highest odds of gestational diabetes, followed by Indian, Sri Lankan, and Pakistani, when compared to US-born white women. Previous studies across the world have suggested 
Table 2 Crude and adjusted odds ratio estimates for adverse perinatal outcomes among South Asian immigrant women with gestational diabetes - NJ births, 1999-2002

\begin{tabular}{|c|c|c|c|c|}
\hline Complications & Non-Hispanic white (referent) & Bangladeshi & Indian & Pakistani \\
\hline \multicolumn{5}{|c|}{ Preterm birth (yes vs no) } \\
\hline Crude & 1.0 & $0.6(0.3-1.1)$ & $0.8(0.7-0.9)$ & $0.7(0.5-0.9)$ \\
\hline Adjusted & 1.0 & $0.6(0.3-1.1)$ & $0.9(0.8-1.1)$ & $0.6(0.4-0.8)$ \\
\hline \multicolumn{5}{|c|}{ Small for gestational age tenth Percentile (yes vs no) } \\
\hline Crude & 1.0 & $3.2(1.9-5.2)$ & $2.6(2.2-2.9)$ & $2.0(1.5-2.8)$ \\
\hline Adjusted & 1.0 & $3.7(2.3-6.0)$ & $2.7(2.3-3.2)$ & $2.2(1.6-3.0)$ \\
\hline \multicolumn{5}{|c|}{ Small for gestational age fifth percentile (yes vs no) } \\
\hline Crude & 1.0 & $2.1(1.0-4.4)$ & $2.7(2.2-3.2)$ & $1.3(0.7-2.2)$ \\
\hline Adjusted & 1.0 & $2.4(1.2-5.1)$ & $2.9(2.3-3.5)$ & $1.5(0.8-2.5)$ \\
\hline \multicolumn{5}{|c|}{ Large for gestational age (birth weight $>4000$ vs $2500-4000 \mathrm{~g}$ ) } \\
\hline Crude & 1.0 & $0.1(0.05-0.5)$ & $0.2(0.1-0.3)$ & $0.6(0.4-0.9)$ \\
\hline Adjusted & 1.0 & $0.2(0.05-0.5)$ & $0.3(0.2-0.3)$ & $0.5(0.3-0.8)$ \\
\hline
\end{tabular}

Sri Lankan women were not included in analysis due to insufficient sample size. All regression models were adjusted for maternal age, education, health insurance coverage, parity, smoking and drinking habits

higher odds of gestational diabetes among overall South Asian immigrant women in USA relative to non-Hispanic white women. Our findings not only further support this phenomenon but also document consistent relatively higher odds of gestational diabetes among immigrants originating from four major South Asian countries.

This study looked into the distribution of risk factors associated with gestational diabetes among immigrants from four major South Asian countries for the first time. In descriptive statistics, we found that Bangladeshi and Pakistani women were less likely to have higher levels of education as well as health insurance coverage compared to Indian and Sri Lankan women. A significant portion of these two immigrants groups also started their prenatal care late compared to others. These three factors are interrelated with each other in a way that lower level of education among immigrants is independently associated with insufficient health insurance coverage leading to inadequate access to health care services, especially in the context of US health care system [16, 17]. Furthermore, acculturation and language barriers of the immigrant population in the host country might be an important predictor of less health care utilization and poor health management $[18,19]$. Although Sri Lankan women, compared to Bangladeshi and Pakistani women, had higher levels of formal education, greater health insurance coverage, and timely initiation of prenatal care, they also had a relatively large number of advanced aged pregnancy and nulliparity, which are two important and independent risk factors of gestational diabetes. These findings support the need for culturally sensitive and language appropriate information and interventions targeting this relevant and numerous group.
When we restricted our analysis to the women with gestational diabetes to compare the odds of pregnancy complications, we found significantly higher odds of having a SGA infant among Indian and Bangladeshi immigrants compared to non-Hispanic whites. Such association of adverse pregnancy outcomes with certain South Asian ethnicity might be explained by absence of preconception screening of risk, late diagnosis of gestational diabetes, and poor health management in these groups. This is especially true for Bangladeshi and Pakistani women who were more likely to start prenatal care in their third trimester (Table 1). The health care continuum framework for gestational diabetes developed by Ohio Gestational Diabetes Collaborative Team that stresses on preconception screening and early diagnosis of gestational diabetes further supports such hypothesis [20]. However, these effects might be a result of confounding by prepregnancy weight or BMI that we could not take into account due to lack of data. Previous studies have identified that maternal thinness is an independent risk factor for preterm birth (gestation age $<37$ weeks) and low birth weight infant [21]. In the absence of data on prepregnancy weight of our study sample, it is very difficult to suggest any possible mechanism underlying this observation. Furthermore, it has been proposed that South Asians have a higher percent of small for gestational age infants compared to mothers of European descent because of physiological differences and the use of standard populationbased growth distribution to determine SGA status [22-24]. This effect might be carried over to the subpopulation with gestational diabetes. The total sample size of Sri Lankan immigrants was very small $(n=193)$ and more research targeting this population is needed in the future. 
The potential limitations of this study include limited availability of data on other risk factors previously reported to be associated with these two conditions. The dataset we used for this study had no information on our study sample's prepregnancy body weight or BMI, family history of gestational diabetes, dietary habits, and physical activity. Furthermore, we considered US-born non-Hispanic whites as a comparison group that is genetically very divergent from South Asians. The difference in risk we observed thus might be explained by genetic predisposition to gestational diabetes among South Asian groups $[25,26]$. US-born women of South Asian ancestry might have been a more appropriate comparison group to foreign born South Asian immigrants than non-Hispanic whites. However due to limited information on the ancestral origins of US-born women in the current dataset, we could not employ this in our analysis. Nonetheless, our analysis could assess the public health burden of gestational diabetes among specific South Asian groups relative to non-Hispanic whites. The major strength of this study includes the analysis of South Asian immigrants as four groups based on their country of origin. Immigration to the US historically has occurred from different South Asian countries under different circumstances, even if these countries are geographically proximal to each other. These immigrants from different countries might differ in their demographic characteristics and health-related behaviors as well as in their access and utilization of health care. We identified for the first time a differential distribution of risk factors, such as maternal age, education, health insurance coverage, parity, and initiation of prenatal care, among immigrants from India, Bangladesh, Pakistan, and Sri Lanka. The second major strength of our study was to use combined birth records and hospital discharge data in case ascertainment of gestational diabetes [5, 13].

Our documented racial disparity in the distribution of risk factors, prevalence, and outcomes of gestational diabetes raises a public health concern. It demands increased resources allotted for the specific South Asian immigrant women. The public health advocacy groups might work toward raising awareness of this condition, early identification of the cases, and appropriate management of the diagnosed cases among this high risk population. Furthermore, a systematic review published in 2002 found the elevated fasting glucose levels during pregnancy to be a significant predictor of type 2 diabetes during the first 5 years after delivery consistently across studies [27]. According to current American Diabetes Association guidelines, the women with gestational diabetes are recommended to undergo glucose screening at 6-8 weeks postpartum and every 3 years thereafter. Our findings warrant more frequent glucose testing and facilitating postpartum preventive behavioral intervention among this high risk immigrant population once they are diagnosed with gestational diabetes in an index pregnancy.

\section{Conclusion and Recommendations}

Our findings present substantial evidence of heterogeneous odds of gestational diabetes among a major immigrant group in New Jersey and disparity in the distribution of some of the associated risk factors as well as subsequent complications in this group comparative to white women. We are among the first to explore the differences in perinatal complications associated with gestational diabetes between women of South Asian origin and non-Hispanic white. These findings recommend stringent blood glucose screening among this high-risk ethnic population and allotment of more resources to ensure preconception risk screening and early initiation of prenatal care. Future research might study the effects of prepregnancy body weight in the association between racial groups and maternal complications related to gestational diabetes as well as more detailed study of insurance status or health care utilization characteristics modifying the risk of gestational diabetes among this immigrant group. Our findings could provide a benchmark for future analyses of more recent data exploring time trends in incidence of gestational diabetes within South Asian immigrant groups at high risk.

Informed Consent No animal or human studies were carried out by the authors for this article. This is a report from secondary data analysis of an existing de-identified database.

Conflict of Interest Sanchalika Acharyya and Teresa Janevic declare that they have no conflict of interest.

\section{References}

1. American Diabetes Association. Diagnosis and classification of diabetes mellitus. Diabetes Care. 2008;31 Suppl 1:S55-60.

2. American Diabetes Association. Diagnosis and classification of diabetes. Diabetes Care. 2007;30 Suppl 1:S42-S47.

3. Ferrara A. Increasing prevalence of gestational diabetes. Diabetes Care. 2007;30(2):5141-6.

4. Thorpe LE, Berger D, Ellis JA, Bettegowda VR, Brown G, Matte T, et al. Trends and racial/ethnic disparities in gestational diabetes among pregnant women in New York City, 1990-2001. Am J Public Health. 2005;95:1536-9.

5. Savitz DA, Janevic TM, Engel SM, et al. Ethnicity and gestational diabetes in New York City, 1995-2003. Br J Obstet Gynaecol. 2008;115:969-078.

6. Dornhorst A, Paterson CM, Nicholls JSD, et al. High prevalence of gestational diabetes in women from ethnic minority groups. Diabet Med. 1992;9:820-5.

7. Chawla A, Amundsen AL, Hanssen KF, et al. Gestational diabetes in women from South East Asia. Tidsskrift Den norske laegeforening. 2006;126(8):1041-3.

8. Carolan MC, Steele C, Margetts H. Attitudes towards gestational diabetes among a multiethnic cohort in Australia. J Clin Nurs. 2010;19(17-18):2446-53. 
9. Ferrarra A, Kahn HS, Quesenberry CP, Riley C, Hedderson MM. An increase in the incidence of gestational diabetes: Northern California, 1991-2000. Obstet Gynecol. 2004;103:526-33.

10. Beischer NA, Oats JN, Henry OA, Sheedy MT, Walstab JE. Incidence and severity of gestational diabetes according to country of birth in women living in Australia. Diabetes. 1991;40(2):35-8.

11. Wu SY. New Jersey's Asian Population by Asian Group: 2010. NJ Department of Labor and Workforce Development. 2012. Retrieved from http://wd.dol.state.nj.us on January, 2013.

12. Gryn T, Gambino C. The foreign born from Asia: 2011. American Community Survey Briefs. 2012. Retrieved from http://www. census.gov/prod/2012pubs/acsbr11-06.pdf on August 2013.

13. Lydon-Rochelle MT, Holt VL, Cardenas V, Nelson JC, Easterling TR, Gardella C, et al. The reporting of pre-existing maternal medical conditions and complications of pregnancy on birth certificates and in hospital discharge data. Am J Obstet Gynecol. 2005;193: 125-34.

14. Oken E, Kleinman K, Rich-Edwards J, Gillman M. A nearly continuous measure of birth weight for gestational age using a United States national reference. BMC Pediatr. 2003;3(1):6.

15. Mongelli M, Wilcox M, Gardosi J. Estimating the date of confinement: ultrasonographic biometry versus certain menstrual dates. Am J Obstet Gynecol. 1996;174(1 Pt 1):278-81.

16. Findings from the CDC Health Disparities and Inequalities ReportUnited States, 2011: Retrieved http://www.cdc.gov/minorityhealth/ reports/CHDIR11/FactSheets/EducationIncome.pdf on August 21 2013.

17. Rabi D, Edwards A, Southern D, Svenson L, Sargious P, Norton P, et al. Association of socio-economic status with diabetes prevalence and utilization of diabetes care services. BMC Health Serv Res. 2006;6:124.
18. Chaudhry S, Fink A, Gelberg L, Brook R. Utilization of Papanicolaou smears by South Asian women living in the United States. J Gen Intern Med. 2003;18:377-84.

19. Venkatesh S, Weatherspoon LJ, Kaplowitz SA, Song WO. Acculturation and glycemic control of Asian Indian adults with type 2 diabetes. J Community Health. 2013;38(1):78-85.

20. Gestational diabetes in Ohio: 2006-2008. Ohio Department of Health report, Oct 2011.

21. Rosenberg T, Garbers S, Lipkind H, Chiasson MA. Maternal obesity and diabetes as risk factors for adverse pregnancy outcomes: differences among 4 racial/ethnic groups. Am J Public Health. 2005;95(9):1545-51.

22. Ray J, Jiang D, Sgro M, Shah R, Singh G, Mamdani M. Thresholds for small for gestational age among newborns of East Asian and South Asian Ancestry. J Obstet Gynaecol Can. 2009;31:322-30.

23. Seaton SE, Yadav KD, Field DJ, Khunti K, Manktelow BN. Birthweight centile charts for South Asian infants born in the UK. Neonatology. 2011;100:398-403.

24. Hanley GE, Janssen PA. Ethnicity-specific birthweight distributions improve identification of term newborns at risk for short-term morbidity. Am J Obstet Gynecol. 2013 ISSN 0002-9378, doi: 10.1016/ j.ajog.2013.06.042.

25. Berkowitz GS, Lapinski RH, Wein R, Lee D. Race/Ethnicity and other risk factors for gestational diabetes. Am J Epidemiol. 1992;135(9):965-73.

26. McKeigue PM, Shah B, Marmot MG. Relation of central obesity and insulin resistance with high diabetes prevalence and cardiovascular risk in South Asians. Lancet. 1991;337:382-6.

27. Kim C, Newton KM, Knopp RH. Gestational diabetes and the incidence of type 2 diabetes: a systematic review. Diabetes Care. 2002;25:1862-8. 